CERN - AB DIVISION

CERN-AB-2003-080 (ABP)

\title{
OBSERVATIONS AND SIMULATION OF A FOURTH ORDER RESONANCE WITH SPACE CHARGE
}

\author{
G. Franchetti ${ }^{1)}$, I. Hofmann ${ }^{1)}$, M. Giovannozzi, M. Martini, and E. Metral
}

\begin{abstract}
A benchmarking experiment with a high intensity bunched beam stored for $1.2 \mathrm{~s}$ in a nonlinear lattice has been performed at the CERN Proton Synchrotron (PS). Beam emittance and beam intensity have been measured for several working points at different distances from a lattice-induced resonance. We found a regime of emittance growth for machine tunes far from the resonance and a regime of emittance shrink/beam loss for working point close the resonance. We compare the observations with 3D simulations and in the blow-up regime we find good agreement. We interpret these results in terms of space charge induced trapping and de-trapping on the lattice resonance. We show that this mechanism is responsible of halo formation which depends on the distance from the resonance and discuss the interplay of dynamic aperture and halo size as main mechanism to explain beam loss in the experiment.
\end{abstract}

Contributed paper at the 29th ICFA Advanced Beam Dynamics Workshop: Beam Halo Dynamics, Diagnostics, and Collimation (HALO'O3), Montauk, Long Island, May 19-23, 2003

Geneva, Switzerland

August 25, 2003

1) GSI, 64291 Darmstadt, Germany 


\title{
Observations and simulation of a fourth order resonance with space charge
}

\author{
G. Franchetti*, I. Hofmann*, M. Giovannozzi ${ }^{\dagger}$, M. Martini ${ }^{\dagger}$ and E. Metral ${ }^{\dagger}$ \\ ${ }^{*}$ GSI, 64291 Darmstadt, Germany \\ ${ }^{\dagger}$ CERN, 1211 Geneva, Switzerland
}

\begin{abstract}
A benchmarking experiment with a high intensity bunched beam stored for $1.2 \mathrm{~s}$ in a nonlinear lattice has been performed at the CERN Proton Synchrotron (PS). Beam emittance and beam intensity have been measured for several working points at different distances from a lattice-induced resonance. We found a regime of emittance growth for machine tunes far from the resonance and a regime of emittance shrink/beam loss for working point close the resonance. We compare the observations with 3D simulations and in the blow-up regime we find good agreement. We interpret these results in terms of space charge induced trapping and de-trapping on the lattice resonance. We show that this mechanism is responsible of halo formation which depends on the distance from the resonance and discuss the interplay of dynamic aperture and halo size as main mechanism to explain beam loss in the experiment.
\end{abstract}

\section{INTRODUCTION}

Up to now space charge effects in beams and singleparticle nonlinear dynamics have always been two distinct areas of interest. Typically, space charge effects have been studied in rings with both simulation and experimental work on the range of thousands of turns [1, $2]$. Resonances in this regime are dominated by space charge. On the contrary, single-particle nonlinear dynamics studies have been carried out in the range of hundred thousand turns (see for instance Ref. [3]). There, nonlinearities are intrinsic to the machine lattice, while selfconsistent effects are absent. For several projects this distinction is natural, as storage times for high-energy machines are long (typically hours), but space charge effects are negligible. In high-intensity machines, however, the beam is stored for much shorter times. This situation allowed an independent development of space charge beam physics and single-particle nonlinear dynamics. However, in new projects such as the SIS100 synchrotron at GSI $[4,5]$ this distinction is no more justified. Storage for $1 \mathrm{~s}$ of a high intensity beam in a nonlinear lattice with loss levels not exceeding $1 \%$ is requested. The interplay between pure single-particle lattice driven nonlinear effects (long-term) and pure space charge effects (short-term) becomes critical. In the simplified simulation model of Ref. [6] it was recently suggested that synchrotron motion induces, because of space charge, a single-particle tune modulation. Consequently, single-particle periodic crossing of a resonance eventually leads to particle trapping into and de-trapping from resonance islands. The synchrotron-space-charge motion induced on resonance islands may take particles in and out the beam core. This process is related to the trapping during a single passage through a higher order nonlinear resonance described in Refs. [7, 8] as a results of a varying tune. In this work we present the results of an experiment performed in a regime of high-intensity beam stored for $1 \mathrm{~s}$ in presence of well- controlled lattice nonlinearity and attempt an interpretation of these results through numerical simulations.

\section{MEASUREMENTS}

The measurements were carried out as part of a high intensity machine development time at the PS in October 2002. The number of protons in the single-bunch (200 ns long at $4 \sigma$ ) was $1.1 \times 10^{12}$, small enough to avoid overlap with other resonances. A vertical maximum space charge tuneshift of 0.12 , and a horizontal one of 0.075 (for minimum amplitude particles) were achieved with relatively small emittances of $\varepsilon_{x}=9 \mathrm{~mm} \operatorname{mrad}$ and $\varepsilon_{y}=$ $4.5 \mathrm{~mm}$ mrad (unnormalized at $2 \sigma$ ). The bunch profiles measured $10 \mathrm{~ms}$ after injection were found to be Gaussian in all directions in the absence of the octupole. The vertical machine tune was set to $Q_{y}=6.12$, and the horizontal tune was varied in the interval $6.25<Q_{x}<6.32$. The chromaticity was close to the natural one, hence the small momentum spread of $10^{-3}$ (at $2 \sigma$ ) allows ignoring chromatic effects. The kinetic energy was kept at the injection value of $1.4 \mathrm{GeV}$ with a measurement window of $1.2 \mathrm{~s}\left(4.4 \times 10^{5}\right.$ turns $)$ over which the bunch intensity was monitored with a current transformer. The calibrated octupole (here $K_{3}=1.215 \times I \mathrm{~m}^{-3}$ ) was powered to $40 \mathrm{~A}$ at $110 \mathrm{~ms}$ after injection to excite the resonance $4 Q_{x}=25$. We used the transverse profiles measured with the flying wire $(20 \mathrm{~m} / \mathrm{s})$, fitted them with a Gaussian profile, and determined the corresponding rms emittances. Initial and 
- in most cases - final profiles were actually found quite close to Gaussian. In Fig. 1 results of final measurements $1.2 \mathrm{~s}$ after injection are plotted as a function of the machine working point.

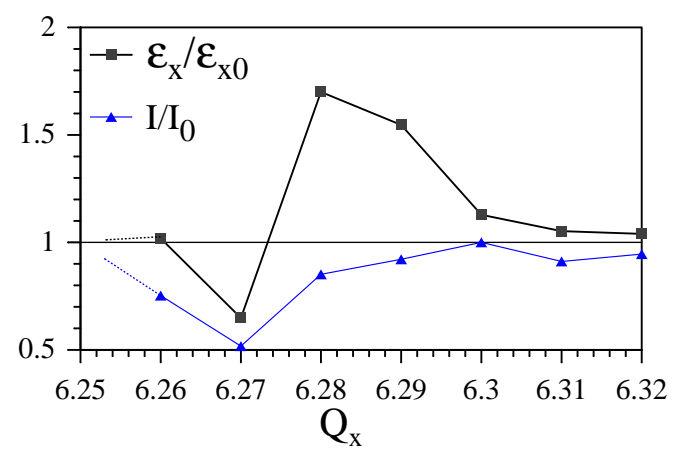

FIGURE 1. Experimental results on final rms emittance (of Gaussian fit) and beam current relative to initial values.

\section{SIMULATION}

Interpretation of these measurements must rely on adequate computer simulations. The comparison is also a necessary basis for code benchmarking on such a long time scale, which has not been undertaken so far to our knowledge. To initiate such a process we have carried out a series of simulation runs in 3D. We have replaced, for simplicity, the linear PS focusing lattice by constant focusing and ignored lattice nonlinearities besides the contribution from the well-defined octupole. We have also ignored the smaller vertical beam emittance of the experiment and assumed a circular cross section to match with the limitation in the analytical 3D space charge model, which is based on a rotational ellipsoid. The horizontal emittance has been re-defined accordingly such as to reproduce accurately the maximum horizontal space charge tuneshift extracted from the measurements, which we believe is the crucial issue here, since we are not dealing with a coupling resonance.

\section{Dynamic aperture}

The loss observed in the experiment must be related to the shrinking of the dynamic aperture, since the beam was too small to hit a physical aperture. To roughly explore the effects on the dynamic aperture of the octupole alone we have carried out a numerical test by searching the maximum stable radius of test particles placed into 20 different directions in the upper half of the $x-y$ plane, and ignoring space charge at this point. We have found that the nominal octupole (40 A) leads to a dynamic aperture $\left(10^{5}\right.$ turns) of about $5 \sigma$ near $Q_{x}=6.25$, where $\sigma$ is the horizontal rms beam size of the injected beam. This value is not small enough to explain the observed extraction of particles as will be seen in the subsequent simulations. Hence, a more complete knowledge of machine nonlinearities at the working points used here may be needed to explain the observed loss. Assuming 200 A octupole current we have calculated that the dynamic aperture shrinks to a radius of $2.5 \sigma$ near $Q_{x}=6.25$ for $10^{3}$ turns, and about $2.2 \sigma$ for $10^{5}$ turns.

\section{D simulations with synchrotron motion}

In [9] it has been found that for a Gaussian beam coherent effects are "Landau-damped". This fact allows to use frozen models for space charge calculation. It was also found in a 2D simulation for $Q_{x}=6.27 \mathrm{using}$ an octupole powered to 200 A that over 3000 turns the emittance increases by $\sim 10 \%$. The failure of $2 \mathrm{D}$ simulation to describe the experiment justifies the need for including the longitudinal motion in a 3D bunch model, which induces a tune modulation primarily via space charge. Space charge is computed using a fully analytical method (see Ref. [10] for more details). It only uses integrals of algebraic expressions in $x, y, z$ and is therefore sufficiently fast and noise-free. We employ a density distribution of the kind $\left(1-x^{2} / a^{2}\right)^{3}$ (in $x$, and similar in $y$ and $z$ ). Its core is sufficiently close to that of a Gaussian, but it has a finite beam edge at $3 \sigma$. Using 2000 test particles we generate the corresponding (initially) consistent distribution in $6 \mathrm{D}$ phase space, assuming the same bunch length ( $200 \mathrm{~ns}$ at $4 \sigma$ ) and synchrotron period (645 turns) as in the experiment.

The dependence of emittance growth on the working point is seen (Fig. 2) to have a similar trend as in the experiment for $Q_{x}>6.28$, but no loss for smaller tunes. For better comparison with the experiment we have applied a Gaussian fit to the simulation data and determined the rms emittance from this fit, which puts the emphasis on the core emittance rather than rms emittances. Note that the relatively large emittance growth without accompanying loss reflects the large physical aperture in both experiment and simulation, if compared with the initial beam size. The resulting maximum halo increases for $Q_{x} \rightarrow 6.25$ (Fig. 3). This is due to the fact that larger betatron amplitudes must be adopted to compensate the increasing space charge, while the particle moves longitudinally to the bunch center and trapping on the resonance island is maintained. Note that for $Q_{x} \rightarrow 6.32$, where the resonance loses its effect, the halo shrinks to the initial beam edge of $3 \sigma$. This halo formation by island trapping is the reason for beam loss in the experiment, where apparently further nonlinearities cause a smaller dynamic aperture than in the simulation and lead to halo extraction. An example of a simulated transverse beam density cut with pronounced halo determined after $5 \times 10^{5}$ turns is shown in Fig. 4 on a logarithmic vertical scale. The to- 


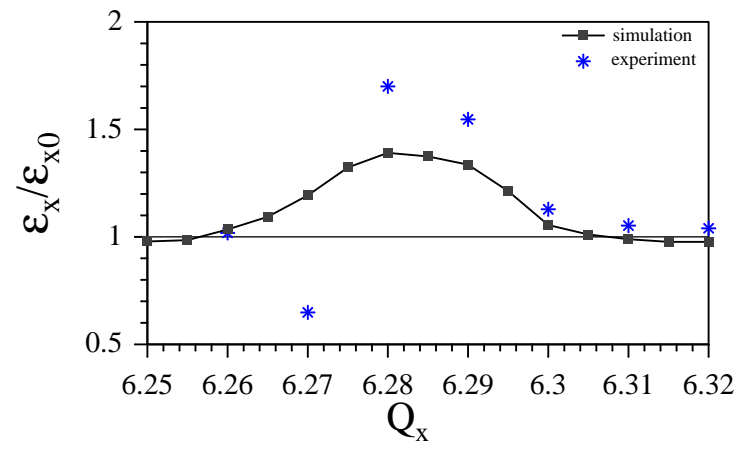

FIGURE 2. 3D simulation using analytical space charge (40 A octupole). Shown are simulated rms emittances (Gaussian fit) after $5 \times 10^{5}$ turns, and experimental values.

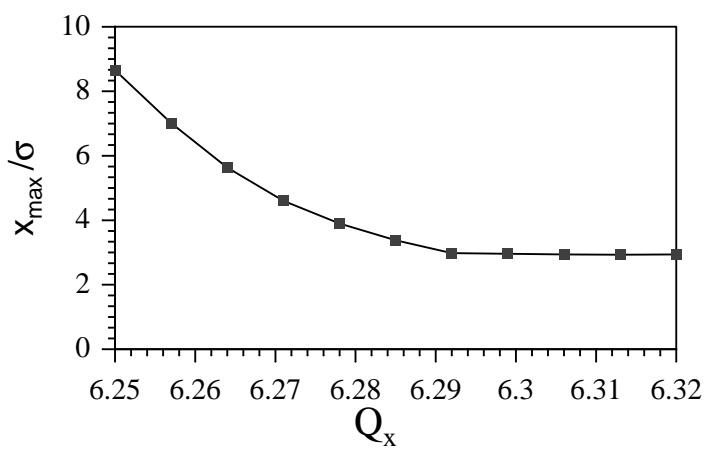

FIGURE 3. Halo radius (in units of initial $\sigma$ ).

tal number of particles in the halo at this instant is about $1 \%$. This quantity does not include the particles, which have been temporarily in the halo at some earlier time, and which would determine the total loss, if a physical aperture would intercept the halo.

\section{Interpretation}

Our interpretation of the significant difference between 2D (only $10 \%$ emittance growth for $\mathrm{I}=200 \mathrm{~A}$, see Ref. [9]) and 3D emittance growth relates this to syn-

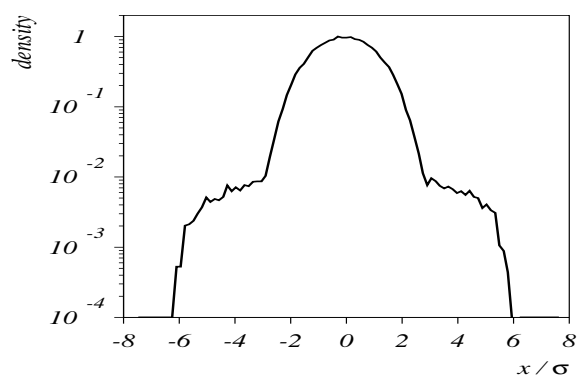

FIGURE 4. Normalized density in simulation for $Q_{x}=6.26$ (radial units in initial $\sigma$ ). chrotron motion: in 2D particles have static tunes and are on resonance for basically one value of the betatron amplitude. Once on resonance they get easily de-tuned again after small amplitude increase due to the dominant space charge detuning; in 3D the synchrotron motion makes the particles oscillate between high and low space charge, which induces an efficient periodic tune modulation. Eventually such particles are locked to the resonance islands, which implies that they may be carried to larger transverse amplitude to compensate the enhanced space charge when moving back to the bunch center. As a single-particle process, hence in the absence of space charge, such amplitude growth by island trapping was studied in detail in Ref. [8], and proposed as beam cleaning method in Ref. [11]. A relatively large number of particles is thus able to cross periodically the resonance at various betatron amplitudes, until trapping occurs. As it was shown in Ref. [6], such trapping may be followed by de-trapping after some time, unless the particle hits the aperture before. This process is shown in Fig. 5 for a single test-particle arbitrarily chosen with maximum synchrotron amplitude, hence initiated at the bunch end. Units on the abscissa are single particle emittances relative to the initial (transverse) beam edge emittance corresponding to $3 \sigma$. The initial relative singleparticle emittance is chosen to be 0.8 and it is plotted at each turn. The tune of $Q_{x}=6.257$ is sufficiently close to the resonance that a large halo radius may be expected, which is confirmed by the simulation. This growth up to 5.5-times the beam edge emittance is consistent with Fig. 3 as it corresponds to $x_{\max } / \sigma=7$. From Fig. 5 (top), we find that the single-particle normalized emittance follows mainly two behaviours: in one it has almost stationary oscillations which last for about half a synchrotron oscillation. The other feature is characterized by emittance jumps. According to our previous description, we attribute such a jump to resonance trapping. In this respect, Fig. 5 (top) suggests that this test particle occasionally gets and remains trapped while going through the bunch center. This in fact corresponds to one oscillation of the single-particle emittance which is a rare event found before and after turn 2000. The most common pattern of the test-particle, however, seems to reveal that trapping and de-trapping happens before the particle passes through the bunch center. For this particular example with $Q_{x}=6.257$ the resonance crossing happens relatively close to the bunch end, justifying therefore the frequency of emittance jump of half synchrotron frequency. In Fig. 5 (top) it is also shown that between two jumps the emittance exhibits almost stationary emittance oscillations. This stems from the combined effect of space charge and octupole nonlinearity. Hence, the larger the particle amplitude the larger the single-particle emittance oscillations. For small emittances, instead, the effect of the octupole is reduced and the emittance os- 

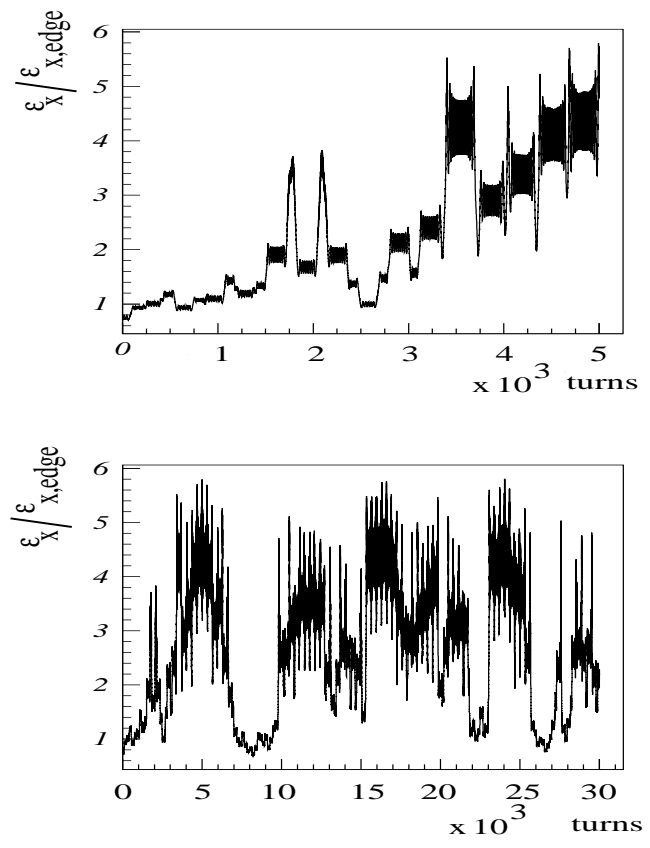

FIGURE 5. Trapping and de-trapping of a single test-particle and $Q_{x}=6.257$ (top frame zoom of bottom frame).

cillations become much smaller. In Fig. 5 (bottom), the single-particle emittance is shown over a longer time scale.

\section{Rms self-consistency}

The simulation rms emittance evolution for $Q_{x}=6.29$ using the fully frozen analytical space charge is shown in Fig. 6. The time profile compares well with the measured data, but saturates somewhat below the experimental values. This saturation is due to the fact that an equilibrium occurs between trapping and de-trapping among the particles with sufficiently large synchrotron amplitude (depending on $Q_{x}$ ) to cross the resonance. We compare this result with a modification, where the growing rms emittance is used to update the horizontal rms size. As a result of this rms self-consistency space charge gets weaker with progressing emittance growth. This allows even particles cross the resonance, which have initially had too small synchrotron amplitudes - correspondingly large tuneshift - to be able to reach the resonance condition. With this increasing number of potentially resonant particles, further emittance growth takes place, and better agreement with the measurement is achieved. While this simple modification works well in the emittance growth regime, it does not help to improve the agreement in the loss regime, where better knowledge of the dynamic aperture is needed.

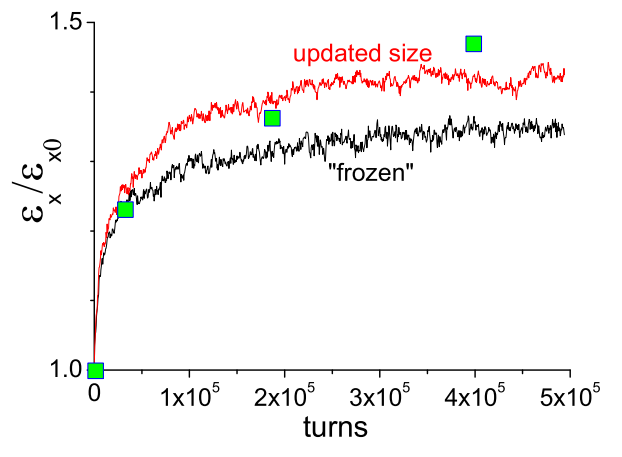

FIGURE 6. 3D simulation emittance evolution for $Q_{x}=6.29$ comparing analytical fully frozen space charge with results obtained by using a continuously updated rms size (squares: measured values).

\section{CONCLUSION}

Synchrotron oscillations have been shown to enhance significantly the effect of the octupole. In the emittance growth regime quite good agreement is achieved with the measurements over half a million turns, which supports our 3D space charge model and the interpretation in terms of tune modulation. We predict the formation of a halo increasing in radius for $Q_{x} \rightarrow 6.25$ and claim this is the source of the measured loss. Future measurements should consider weaker octupoles, where the predicted halo might be entirely inside the dynamic aperture and observable by scrapers. Cross-checks with fully selfconsistent 3D simulation over some $10^{4}$ turns (stronger octupole case) are planned for the near future.

\section{REFERENCES}

1. S. Cousineau et al., Phys. Rev. ST Accel. Beams 6, 034205 (2003).

2. K. Takayama et al., EPAC02, p. 1413 (2002).

3. M. Giovannozzi, this conference.

4. W. Henning, Proceedings of the 2003 Particle Accelerator Conference, Portland, Or., USA (2003).

5. P. Spiller et al., ibid.

6. G. Franchetti and I. Hofmann, AIP Conf. Proc. 642, 260 (2002).

7. A. Schoch, CERN Report, CERN 57-23 (1958).

8. A.W. Chao and M. Month, Nucl. Instr. and Meth. 121, 129 (1974).

9. I. Hofmann et al., this conference.

10. G. Franchetti, I. Hofmann, M. Giovannozzi, M. Martini, E. Metral, to be submitted to Phys. Rev. ST Accel. Beams.

11. A.W. Chao and M. Month, Nucl. Instr. and Meth. 133, 405 (1976). 